\title{
Design and Verification of Outbound Mode Strategy Based on EIQ Analysis
}

\author{
Shuo Wang, Daofang Chang, Zhenyu Xu \\ Shanghai Maritime University, Shanghai, China \\ Email: 17862826195@163.com
}

How to cite this paper: Wang, S., Chang, D.F. and Xu, Z.Y. (2019) Design and Verification of Outbound Mode Strategy Based on EIQ Analysis. American Journal of Industrial and Business Management, 9, 16251638.

https://doi.org/10.4236/ajibm.2019.98106

Received: July 4, 2019

Accepted: August 9, 2019

Published: August 12, 2019

Copyright $\odot 2019$ by author(s) and Scientific Research Publishing Inc. This work is licensed under the Creative Commons Attribution International License (CC BY 4.0).

http://creativecommons.org/licenses/by/4.0/

\begin{abstract}
In this paper, in view of the poor delivery efficiency of the warehouse, the theoretical analysis and mathematical modeling are closely linked by collecting and statistics data of a large number of data from single storehouse and single warehouse. The qualitative analysis and quantitative calculation are combined with a large number of actual data obtained by actual operation. Based on this, a discriminant model of the outgoing mode based on EIQ analysis is established to solve the data into the model. It can be seen from the verification results that the error of the time difference of the way out of the warehouse can be less than $\pm 25 \mathrm{~s}$, and it can be proved that the model is practical and reasonable in the selection decision for the way of out of the storehouse, and can provide reference for the selection of a batch order. Finally, the optimized scheme of the outlet of the solid warehouse is obtained, which improves the warehouse outgoing efficiency.
\end{abstract}

\section{Keywords}

EIQ Analysis, The Discriminant Model of Outbound Mode, Flexsim Simulation, Improvement of Facilities and Equipment, Outbound Efficiency

\section{Introduction}

After investigation of the logistics distribution center, after re-planning the traveling route of the stacker crane and the related strategy of using the partitioned storage, these practices have improved the operating efficiency of the stacker crane in the three-dimensional warehouse, but there are still many problematic limitations. The warehouse efficiency of a three-dimensional warehouse, for example:

1) After the RGV trolley is running, the speed is slower. Selecting the goods to be reassembled and then passing through the RGV trolley can be stored in the 
warehouse, resulting in a very low effective delivery.

2) If a warehouse is single-selected and warehoused, the number of operations will be many, it will take a long time and the efficiency will be low. If the combined orders are shipped out, although the number of operations is reduced, secondary sorting is also required using the wave sorting system, which increases costs. Therefore, for automated warehouse, there is no effective way to identify the outgoing mode.

Based on the above reasons, this paper according to the actual operation of the logistics distribution center, analyzes and explores the efficiency of the warehouse out of the warehouse in the course of the actual operation of the logistics, and takes the EIQ strategy as the theoretical basis for the solution. At the same time, the mathematical model is set up according to the data obtained by the EIQ analysis. After modeling, it is verified in the virtual central system.

The structure of the article is as follows. The introduction part explains the reason why the warehouse of the three-dimensional warehouse in the logistics distribution center is not efficient. Then the first part of the problem description introduces the key to solving the efficiency of the outbound library area. The EIQ analysis is used to establish the judgment model of the outbound method, so that the appropriate outbound method can be selected according to different orders, and the outbound operation time can be reduced. In the second part, based on the data obtained from the EIQ analysis of the order information, a judgment model based on EIQ analysis is established. In order to prove the rationality and practicability of the model, the third part verifies the correctness of the model by substituting the actual order information. The final conclusions show that the research improves the warehouse outbound efficiency and the service quality of the enterprise, which can provide certain reference value for the practical problem solving of the enterprise.

\section{Problem Description}

How to improve the efficiency of warehouse picking operations for three-dimensional warehouses, mainly to select the appropriate way out of the library, reduce the time of outbound operations and other aspects began to analyze. According to the analysis of the current warehouse and the running characteristics of the warehouse, it is found that the key to the efficiency of the three dimensional reservoir area is how to effectively reduce the time of the outgoing operation.

After comparing various methods for reducing work time, it was found that combining EIQ analysis to establish a judgment model is the most suitable method to solve this problem. The specific operation is to select an order within a certain period of time according to the window-by-window batch method, and to test the order of the time period, and compare the two orders of delivery method: single order delivery and combined order delivery.

The analysis and test results show that the two delivery methods are applicable to different types of orders, rather than the absolute type of high efficiency. 
Therefore, the selection of the delivery method should be selected based on the characteristics of the order within the time period. For the analysis of the order characteristics, the most commonly used is the EIQ analysis, that is, the analysis of the quantity of the order items. Use the three logistics key elements " $\mathrm{E}$ " (order), "I" (item item), and "Q" (quantity) analyze the order, thus providing the basis for the choice of the way out of the library [1]. Based on the data obtained from the EIQ analysis and combined with the time data obtained from the test in the virtual simulation system, an abstract simplified model was established to determine the specific order of a lot of orders.

\section{Mathematical Model}

\subsection{Model Construction Analysis}

Orders within a certain period of time are suitable for single-order delivery or combined order delivery. They need to be selected according to the characteristics of the order within the time period. Therefore, in order to increase the efficiency of the logistics center, it is necessary to first check the period of time. The characteristics of the internal orders are analyzed, and the most efficient outbound delivery method is selected based on the analysis results.

The time required for each step out of the warehouse is related to the order items, order quantity, and other information. Assuming that the worker's operational proficiency is fixed, only the order information is needed to determine the optimal shipping method and the fastest shipping time. Based on the data obtained from the EIQ analysis of the orders, a multivariate functional relationship was established to associate the order information with the time spent in the warehouse and a certain relationship was established between the two. As long as you know the details of the order, you can use this function to get the time required for the delivery.

In terms of the characteristics of shipments, logistics centers often have features such as indefinite shipping time, short lead times, and large changes in shipments. Traditionally used methods for total and average analysis have often resulted in large differences from actual results. By establishing an EIQ analysis model, the principles of data analysis can be mastered, and through effective data statistics and further related analysis, the process of analysis can be simplified and actual useful information can be grasped. To provide a data base for establishing the shipping mode judgment model [2].

1) Model assumption:

a) Items of all orders are stored in the three-dimensional library area.

b) The operational proficiency of each staff member is not affected by the external environment.

c) Each the same time the goods arrive at the port of delivery from the logistics area.

d) The outbound goods are in the smallest unit.

2) Symbol Description (Table 1) 
Table 1. Symbol interpretation table.

\begin{tabular}{cc}
\hline Symbol & Significance \\
\hline$E_{m}(m=1,2,3 \ldots)$ & Orders \\
$I_{n}(n=1,2,3 \ldots)$ & Food items \\
$Q_{m n}$ & The number of nth orders for the $m$ th order \\
$N$ & Total shipment items \\
GN & The number of orders shipped items accumulated times \\
\hline
\end{tabular}

\section{3) Data acquisition and processing}

a) Determination of the time range for order batching:

When conducting the EIQ analysis, first of all, the time range and unit must be considered. According to the time-window batch method principle, orders in one day are batched. From the operation data analysis of the logistics center, we know that the number of daily operation orders Etotal in the logistics center is approximately 130 . The daily working hours are from 8 to 19:00 and the total time $T$ is 11 hours. Too many batches, the processing is troublesome and batch results are not too large meaning; too few, there are more orders per batch, and the order waits longer.

- If the time window is set to 40 minutes, the daily order divided into 16.5 batches, with an average of 7.879 sheets per batch single.

- If the time window is set to 30 minutes, the daily order divided into 22 batches with an average of 5.909 orders per batch.

- Calculate If the time window is set to 20 minutes, the daily orders are divided there are 33 batches with an average of 3.939 orders per batch.

- From the analysis of the calculation results, we can see that the time window is set to 40 minutes, and there are many orders for each batch. It is troublesome to process the orders. Set the time window to 20 minutes, the batch in one day the number of times reached 33 batches and the number was larger.

- Through the statistical analysis of the order data and the actual operation experience of the workers, the time window is set to 30 minutes after comprehensive consideration, that is, every 30 minutes to receive a batch of orders.

b) EIQ database statistics: Assume that from 8 o'clock to 8:30pm. The flow center received a number of orders, and statistics were made on the order information of the first batch. Outbound orders $E_{m}(m=1,2,3, \cdots)$ were included in the batch. There are shipping items involved in all orders $I_{n}(n=1,2,3, \cdots)$ The $Q_{m n}=$ Quantity (Order $E_{m}$, Item $I_{n}$ ) symbol represents the number of items ordered by a single order [3]. The statistics of the orders are plotted in a table, as shown in Table 2.

Based on the above analysis results, the following analysis can be carried out. From the data in Table 2, the following types of analysis can be performed:

- Order Quantity (EQ) Analysis: Single-sheet order quantity analysis [5]. 
Table 2. EIQ statistics for the first batch of orders on a certain day.

\begin{tabular}{|c|c|c|c|c|c|c|c|c|}
\hline \multirow{2}{*}{ Shipping order } & \multicolumn{6}{|c|}{ Shipment items } & \multirow{2}{*}{$\begin{array}{c}\text { Order } \\
\text { shipment } \\
\text { quantity }\end{array}$} & \multirow{2}{*}{$\begin{array}{l}\text { Order } \\
\text { items }\end{array}$} \\
\hline & $I_{1}$ & $I_{2}$ & $I_{3}$ & $I_{4}$ & $\ldots$ & $I_{n}$ & & \\
\hline$E_{1}$ & $Q_{11}$ & $Q_{12}$ & $Q_{13}$ & $Q_{14}$ & & $Q_{1 n}$ & $D Q_{1}$ & $N_{1}$ \\
\hline$E_{2}$ & $Q_{21}$ & $Q_{22}$ & $Q_{23}$ & $Q_{24}$ & & $Q_{2 n}$ & $D Q_{2}$ & $N_{2}$ \\
\hline$E_{3}$ & $Q_{31}$ & $Q_{32}$ & $Q_{33}$ & $Q_{34}$ & & $Q_{3 n}$ & $D Q_{3}$ & $N_{3}$ \\
\hline$E_{4}$ & $Q_{41}$ & $Q_{42}$ & $Q_{43}$ & $Q_{44}$ & & $Q_{4 n}$ & $D Q_{4}$ & $N_{4}$ \\
\hline \multicolumn{9}{|l|}{$\ldots$} \\
\hline$E_{m}$ & $Q_{m 1}$ & $Q_{m 2}$ & $Q_{m 3}$ & $Q_{m 4}$ & & $Q_{m n}$ & $D Q_{m}$ & $N_{m}$ \\
\hline $\begin{array}{l}\text { Single product } \\
\text { shipment }\end{array}$ & $C Q_{1}$ & $C Q_{2}$ & $C Q_{3}$ & $C Q_{4}$ & & $E_{m}$ & $Q$ & $N$ \\
\hline $\begin{array}{l}\text { Single product } \\
\text { shipments }\end{array}$ & $K_{1}$ & $K_{2}$ & $K_{3}$ & $K_{4}$ & & $K_{m}$ & - & $K[4]$ \\
\hline
\end{tabular}

- Analysis of the number of ordered items (EN): Analysis of the number of single-order items shipped.

- Item Quantity (IQ) Analysis: Analysis of the total quantity shipped for each item.

- Analysis of Item Acceptance (IK): analysis of the number of shipments per single item [6].

- According to the purpose of the EIQ analysis of the order, the analysis of the order item number (EN) is beneficial to the selection of the exporter of the batch of orders [7].

c) Analysis of the number of items ordered (EN): The analysis of the number of items of orders (EN) mainly understands the distribution of the number of items ordered for each order, has a great influence on the principles of order processing and the planning of the picking system, and will affect the planning of shipping methods and shipping areas. It is usually necessary to coordinate with the three indicators of the total number of items shipped, the cumulative number of items shipped items and the total number of items.

\subsection{Establishing the Outbound Mode Judgment Model}

\section{1) Adopt single-by-one method:}

According to Gantt chart analysis and the process of single outbound operations, the total time required to complete a single order for a single batch of orders is $T_{d}$ mainly includes order processing time $T_{1}$, stereo shelf operation time $T_{2}$, cargo removal time $T_{3}$, review, packaging, labeling and handling time $T_{4}$.

$$
T_{d}=\min \left(T_{1}+T_{2}+T_{3}+T_{4}\right)
$$

a) Order processing time $T_{1}$ : In the virtual simulation system, the processing time of an order is tested. From the time the computer is started to leave the office for processing time, the average value is calculated after five tests. The test data is shown in Table 3. 
Table 3. Processing time of an order.

\begin{tabular}{ccccccc}
\hline Number of trials & $\mathbf{1}$ & $\mathbf{2}$ & $\mathbf{3}$ & $\mathbf{4}$ & $\mathbf{5}$ & Average value \\
\hline Time $(\mathrm{s})$ & 59 & 62 & 65 & 68 & 64 & 63.6 \\
\hline
\end{tabular}

Calculate that the average processing time of an order is $t_{1}=63.6 \mathrm{~s}$ and the order processing time is

$$
T_{1}=m \cdot t_{1}
$$

b) Stereoscopic shelf operating time $T_{2}$ : Stereoscopic shelf operation time $T_{2}$ is the sum of the stereo shelf life of all orders.

$$
T_{2}=\sum t_{2} m(m=1,2,3, \cdots)
$$

Testing each order of three-dimensional shelves in a virtual simulation system running time $t_{2} m$, starting from the stereo racking work to the RGV trolley delivering the trays to the tray opening, plus the time for the goods to be sent back to the stereoscopic storage area for the three-dimensional shelf after the disc removal is completed, excluding the time for the cargo to be removed. Assuming that each cargo arrives at the same time from the cargo space to the cargo port, the cubic shelf operation $t_{2} m$ of the $m$-th order $E_{m}$ is related to the number of items $N_{m}$ of the order. Add five orders for testing by yourself. Test data and results are shown in Table 4.

From the test data, it can be seen that the running time of an order's three-dimensional shelf has a linear relationship with the number of items shipped in the order.

$$
t_{2} m=a_{1} \cdot N_{m}+b_{1}
$$

According to the data obtained from the test, a linear regression method was used to fit the data. The parameters $a_{1}=72$ and $b_{1}=44.4$ were calculated. The fitting curve is shown in Figure 1.

c) Cargo removal time $T_{3}$ : In the virtual simulation system, test the time required for disc removal $T_{3}$, and add the order for five experiments. The total shipments in the five experiments were set to $5,10,15,20$, and 25 boxes. The test results are shown in Table 5.

In the case of constant operator proficiency, the goods are removed time is approximately linear with total shipments

$$
T_{3}=a_{2} \cdot Q+b_{2}
$$

Fit a linear regression to get the fit of Figure 2. $a_{2}=7.1, b_{2}=1.7$

d) Review, packing, labeling and handling time $T_{4}$ : Tests are carried out in a virtual simulation system, from the time when the goods are transported to the recheck port through the conveyor belt and the storage cage is pushed to the exit port for a time of review, packing, labeling and handling. Add orders by yourself and conduct 5 experiments. The test data is shown in Table 6 .

According to the operation flow and actual test data analysis, the review, packing, labeling, and handling time $t_{4}$ of each order is approximately linear with the order shipment $D Q_{n}$. 
Table 4. Stereo shelf run time for an order.

\begin{tabular}{cccccc}
\hline Order number & $\mathbf{1}$ & $\mathbf{2}$ & $\mathbf{3}$ & $\mathbf{4}$ & $\mathbf{5}$ \\
\hline $\begin{array}{c}\text { Order items } \\
\text { Stereoscopic shelf operation Time (s) }\end{array}$ & 1 & $\mathbf{2}$ & $\mathbf{3}$ & $\mathbf{4}$ & $\mathbf{5}$ \\
\hline
\end{tabular}

Table 5. Deconsolidation time for different total shipments.

\begin{tabular}{cccccc}
\hline Experiment times & $\mathbf{1}$ & $\mathbf{2}$ & $\mathbf{3}$ & $\mathbf{4}$ & $\mathbf{5}$ \\
\hline Total shipments & 5 & 10 & 15 & 20 & 25 \\
Time $(\mathrm{s})$ & 46 & 67 & 104 & 134 & 190 \\
\hline
\end{tabular}

Table 6. Time for reviewing, packing, labeling, and handling an order.

\begin{tabular}{cccccc}
\hline Order number & $\mathbf{1}$ & $\mathbf{2}$ & $\mathbf{3}$ & $\mathbf{4}$ & $\mathbf{5}$ \\
\hline Order shipments & 2 & 4 & 6 & 8 & 10 \\
Time $(\mathrm{s})$ & 117 & 134 & 157 & 179 & 212 \\
\hline
\end{tabular}

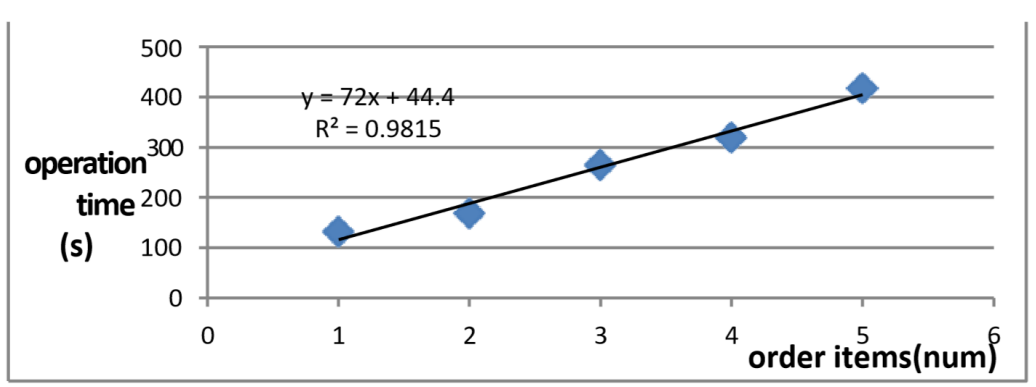

Figure 1. Three-dimensional shelf life time chart of an order.

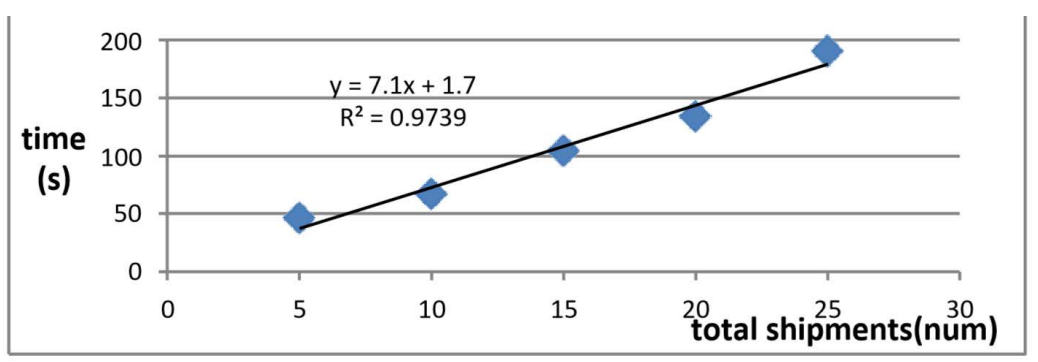

Figure 2. Fitting chart for the time of disc removal and total shipment.

$$
t_{4}=a_{3} \cdot D Q_{n}+b_{3}
$$

According to the test data, the method of linear regression is used fit the line, and the fit is shown in Figure 3. Calculate the parameter $a_{3}=11.75, b_{3}=89.3$.

Since the review, packing, labelling, and handling of each order starts, the next order has already started order processing and shipment from the three-dimensional warehouse, so the total review, packaging, labeling, and handling time $T_{4}$ only includes Last order's operation time.

$$
T_{4}=t_{4}
$$




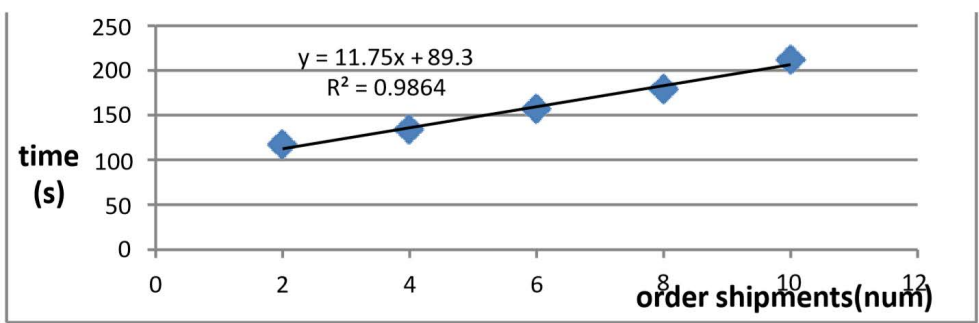

Figure 3. Review of packaging, labeling, and handling time and shipments of the review.

\section{2) Merge orders outbound operations:}

According to the analysis of Gantt chart and the flow of combined order outbound operations, the total time required for the completion of a single batch of orders using the consolidated order is $T_{h}$, which mainly includes the order processing time $T_{1}$, and the three-dimensional shelf operation time $T_{2}$, Cargo Removal Time $T_{3}$, Wave Sorting System Time $T_{5}$, Review, Packing, Labeling and Handling Time $T_{4}$.

$$
T_{h}=\min \left(T_{1}+T_{2}+T_{3}+T_{4}+T_{5}\right)
$$

a) Order processing time $T_{1}$ : When using the merge order picking method, the order processing time $T_{1}$ of the consolidated order is tested in the virtual simulation system. Orders were added for five experiments on their own. The number of consolidated orders in each experiment was $2,3,4,5$, and 6 , the test data is shown in Table 7.

According to the operation flow and experimental test data analysis, order processing the time $T_{1}$ is approximately linear with the order number $m$

$$
T_{1}=a_{4} m+b_{4}
$$

Fit the data obtained from the test. As shown, calculate the parameter $a_{4}=5.4$, $b_{4}=61.2$ (Figure 4).

b) Stereoscopic shelf operating time $T_{2}$ : When using the combined order picking method, the wave order is regarded as an order for one time out of storage. Therefore, the three-dimensional shelf operation time $T_{2}$ is linearly related to the total item number $N$. The analysis shows that the time and the press release The data tested in the library has the same rule, ie the parameters $a_{1}$ and $b_{1}$ are the same.

$$
T_{2}=a_{1} N+b_{1}
$$

Parameter $a_{1}=72, b_{1}=44.4$

c) Cargo removal time $T_{3}$ : With the proficiency of the operator remaining unchanged, the time for the demolition of the goods is approximately proportional to the total shipments. Since the total shipments have not changed, the time for the demolition of the goods is the same as the time for the demolition of the goods in the single picking mode.

$$
T_{3}=a_{2} \cdot Q+b_{2}
$$

Parameter $a_{2}=7.1, b_{2}=1.7$. 
Table 7. Consolidation order processing time.

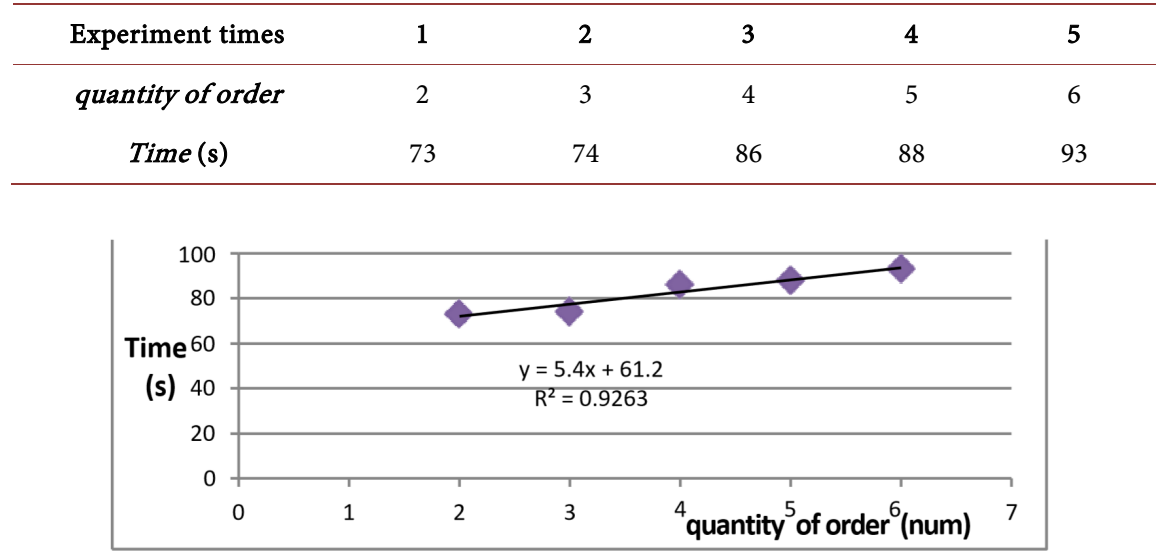

Figure 4. Merge order processing time curve.

According to the analysis of Gantt chart and the flow of combined order outbound operations, the total time required for the completion of a single batch of orders using the consolidated order is $T_{h}$, which mainly includes the order processing time $T_{1}$, and the three-dimensional shelf operation time $T_{2}$, Cargo Removal Time $T_{3}$, Wave Sorting System Time $T_{5}$, Review, Packing, Labeling and Handling Time $T_{4}$.

d) Wave sorting system time $T_{5}$ :

$$
T_{5}=\sum t_{5 n}
$$

The time for the wave sorting of the cargo of item $n$ is $t_{5 n}$, this time has the following relationship with the shipment $C Q_{n}$ and the number of shipments $K_{n}$ of the item:

$$
t_{5 n}=a_{5} \cdot C Q_{n}+b_{5} \cdot K_{n}+c_{5}
$$

To the determination of parameters $a_{5}, b_{5}$, and $c_{5}$ Value, using the control variable method. First set the single product shipment times $K_{n}$ as the fixed value, change the value of single product shipment $C Q_{n}$, add the order information by itself, and test the single product wave sorting time in the virtual simulation system $t_{5 n}$. The test result data is shown in Table 8.

According to the test data for linear regression fitting, the fitting diagram is shown in the Figure 5.

As shown in Figure 5, find the parameter $a_{5}=9.27$

After that, the single product shipment $C Q_{n}$ is set as a value, and the value of single product shipment times $K_{n}$ is changed, and the single product wave sorting time $t_{5 n}$ is measured. The test result data is shown in Table 9.

According to the test data for linear regression fitting, the fitting diagram is shown in Figure 6, to solve the parameters $b_{5}=25.5, c_{5}=24$.

e) Review, packing, labeling and handling time $T_{4}$ :

After the operation of the wave sorting system is completed, the operations of review, packing, labeling, and handling are performed. The time $T_{4}$ is proportional to the order quantity $m$. 
Table 8. Control item shipment times wave sorting time.

\begin{tabular}{ccccc}
\hline items & Super rice cooker & Voltage cooker & Smart rice cooker & IH rice cooker \\
\hline $\begin{array}{c}\text { Single product } \\
\text { shipment times }\end{array}$ & 4 & 4 & 4 & 4 \\
$\begin{array}{c}\text { Single product } \\
\text { shipments }\end{array}$ & 4 & 8 & 16 & 24 \\
Time (s) & 129 & 162 & 235 & 314 \\
\hline
\end{tabular}

Table 9. Control item shipment wave sorting time.

\begin{tabular}{ccccc}
\hline items & Super rice cooker Voltage cooker & Smart rice cooker & IH rice cooker \\
\hline $\begin{array}{c}\text { Single product } \\
\text { shipment times }\end{array}$ & 1 & 2 & 3 & 4 \\
$\begin{array}{c}\text { Single product } \\
\text { shipments }\end{array}$ & 4 & 4 & 4 & 4 \\
Time (s) & 52 & 68 & 107 & 124 \\
\hline
\end{tabular}

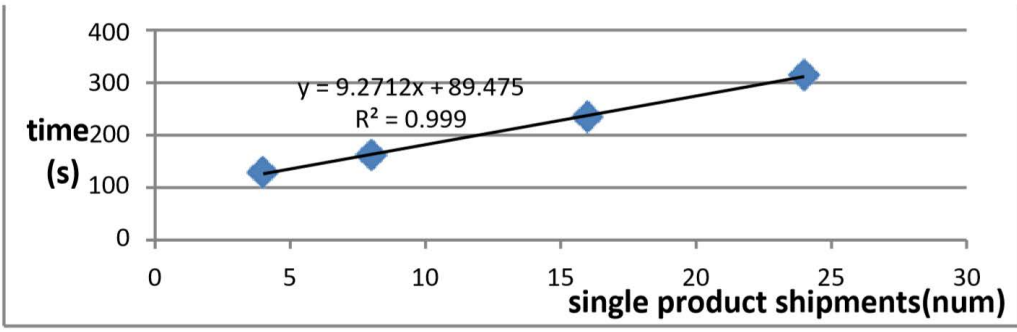

Figure 5. Control of single product shipment times wave sorting time plot.

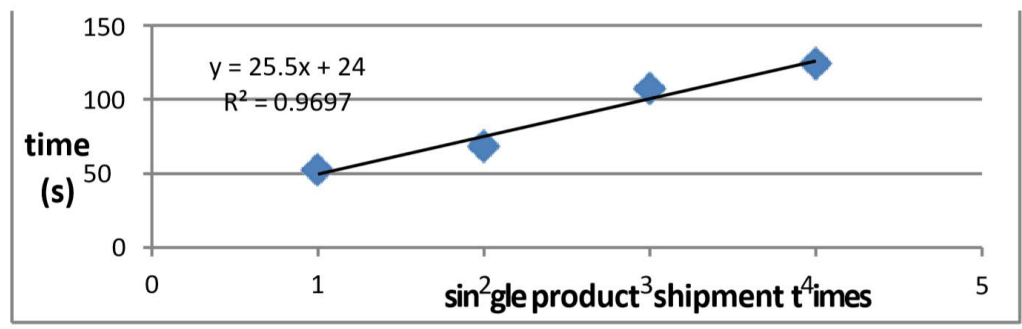

Figure 6. Control item shipments wave sorting time fit graph.

$$
T_{4}=a_{6} \cdot m
$$

Add order information by oneself and test the verification, packing, labeling and handling time $T_{4}$ in the virtual simulation system, each experiment there are $1,2,3,4$, and 5 orders placed in the order. Test result data Table 10 shows.

According to the test results, a linear regression fit was performed, and the intercept of the regression line equation was set to zero. The fitting diagram is shown in Figure 7, with the parameter $a_{6}=70.873$.

\section{3) Objective function:}

The shortest outbound time $T$ for a batch order is the shortest of the two delivery methods. 
Table 10. Review, package, labeling, and handling times for different order quantity.

\begin{tabular}{cccccc}
\hline Experiment times & $\mathbf{1}$ & $\mathbf{2}$ & $\mathbf{3}$ & $\mathbf{4}$ & $\mathbf{5}$ \\
\hline quantity of order & 1 & 2 & 3 & 4 & 5 \\
Time $(s)$ & 78 & 162 & 201 & 287 & 349 \\
\hline
\end{tabular}

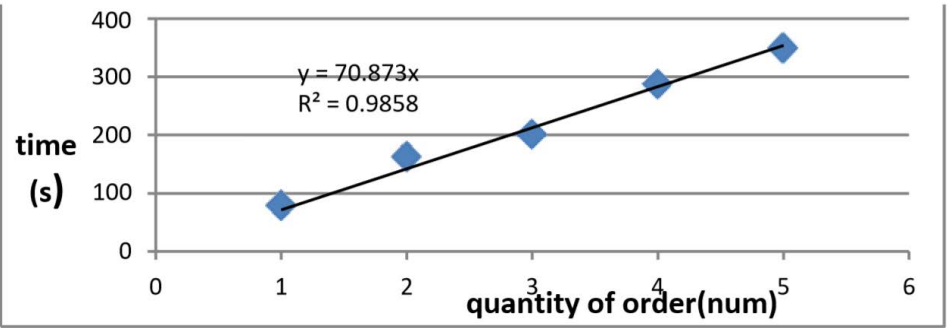

Figure 7. Review of the review, packaging, labeling, and handling time.

$$
T=\min \left(T_{d}, T_{h}\right)
$$

Comparing the sizes of $T_{d}$ and $T_{h}$, it can be judged which one is out of stock the efficiency of the method is higher, and the proper way of picking out the goods according to the characteristics of the order is achieved.

\section{Outbound Mode Judgment Model Test}

The outbound mode judgment model is based on the time-separation and summation of the job flow, and a simplified mathematical model of the actual job flow is obtained. The parameters of the model are calculated based on the data obtained from multiple experiments. In order to prove the rationality and practicality of the model, the correctness of the model is verified by substituting the actual order information.

\subsection{Pre-Recorded Order Inspection Analysis}

Four orders pre-recorded by the system are subjected to EIQ analysis. The results of the analysis are shown in Table 11.

Known order quantity: $m=4$, item quantity: $n=4$, total shipment quantity: $Q$ $=44$, total shipping item item number: $N=4$, total item quantity of all items: $K$ $=14$, accumulated shipment quantity Number of items: $G N=14$. Substituting the data obtained by the analysis into the outbound mode judgment model.

1) Single outbound inspection

Order processing time $T_{1}$ :

$$
T_{1}=m \cdot t_{1}=4 \times 63.6=254.4(\mathrm{~s})
$$

Stereoscopic shelf operating time $T_{2}$ :

$$
T_{2}=\sum t_{2 m}=\sum a_{1} \cdot N_{m}+b_{1}=72 \times 14+44.4 \times 4=1185.6(\mathrm{~s})
$$

Cargo removal time $T_{3}$ :

$$
T_{3}=a_{2} \cdot Q+b_{2}=7.1 \times 44+1.7=314.1(\mathrm{~s})
$$


Table 11. EIQ statistics table for 4 orders pre-recorded by the system.

\begin{tabular}{ccccccc}
\hline \multirow{2}{*}{$\begin{array}{c}\text { Shipping order } \\
\text { number }\end{array}$} & $\begin{array}{c}\text { Super rice } \\
\text { cooker }\end{array}$ & $\begin{array}{c}\text { Voltage } \\
\text { cooker }\end{array}$ & $\begin{array}{c}\text { Smart rice } \\
\text { cooker }\end{array}$ & $\begin{array}{c}\text { IH rice } \\
\text { cooker }\end{array}$ & Export quantity & $\begin{array}{c}\text { Shipment } \\
\text { items }\end{array}$ \\
\hline 1 & 2 & 1 & 2 & 6 & 11 & 4 \\
2 & 3 & 2 & 0 & 6 & 11 & 3 \\
3 & 1 & 1 & 2 & 7 & 11 & 3 \\
4 & 3 & 0 & 3 & 5 & 11 & 4 \\
Shipments & 9 & 4 & 7 & 24 & 44 & 4 \\
\hline
\end{tabular}

Review, packing, labeling and handling time $T_{4}$ :

$$
T_{4}=t_{4}=a_{3} \cdot D Q_{n}+b_{3}=11.75 \times 11+89.3=218.55(\mathrm{~s})
$$

By single delivery time $T_{d}$

$$
T_{d}=\min \left(T_{1}+T_{2}+T_{3}+T_{4}\right)=1972.65(\mathrm{~s})
$$

\section{2) Merge orders checkout method}

Order processing time $T_{1}$ :

$$
T_{1}=a_{4} m+b_{4}=5.4 \times 4+61.2=82.8(\mathrm{~s})
$$

Stereoscopic shelf run time $T_{2}$ :

$$
T_{2}=a_{1} N+b_{1}=72 \times 4+44.4=332.4(\mathrm{~s})
$$

Cargo Removal Time $T_{3}$ :

$$
T_{3}=a_{2} \cdot Q+b_{2}=7.1 \times 44+1.7=314.1(\mathrm{~s})
$$

Wave sorting time $T_{5}$ :

$$
\begin{aligned}
T_{5} & =\sum t_{5 n}=\sum\left(a_{5} \cdot C Q_{n}+b_{5} \cdot K_{n}+c_{5}\right) \\
& =209.43+137.58+165.39+348.48=860.88(\mathrm{~s})
\end{aligned}
$$

Review, packing, labeling and handling time $T_{4}$ :

$$
T_{4}=a_{6} \cdot m=70.873 \times 4=283.492(\mathrm{~s})
$$

Consolidated order leaving time $T_{h}$ :

$$
T_{h}=\min \left(T_{1}+T_{2}+T_{3}+T_{4}+T_{5}\right)=1873.672(\mathrm{~s})
$$

According to the calculation results, the combined order delivery time $T_{h}$ to be slightly less than the single delivery time $T$, so choose to merge orders out the method is more efficient.

\subsection{Analysis of Model Test Results}

The time calculated using the model is compared with the data obtained from the actual experiment. The results of the comparison are shown in Table 12.

From Table 12, it can be seen that although the time used to calculate the time 
Table 12. Comparison between the time calculated by the four pre-recorded order models and the actual time.

\begin{tabular}{ccccc}
\hline & $\begin{array}{c}\text { Calculate the time data Actual experimentally } \\
\text { using the model }\end{array}$ & Time error & Error rate \\
\hline $\begin{array}{c}\text { Single delivery time } \\
\text { merge with time }\end{array}$ & 1972.65 & 1721 & 251.65 & $14.62 \%$ \\
$\begin{array}{c}\text { of delivery } \\
\text { Time difference } \\
\text { of delivery }\end{array}$ & 1873.672 & 1645 & 228.67 & $13.90 \%$ \\
\hline
\end{tabular}

spent and the actual life there is a certain degree of difference, about $15 \%$, but the use of this model to calculate the results of the difference between the library time is realistic, the gap About $25 \%$.

The main reason for the error rate of $30.23 \%$ for the time difference between the four types of orders for the pre-recorded orders of the system is: the time difference between the two types of orders for this batch of orders is not significant.

The time difference of the way out of the warehouse is within $\pm 25 \mathrm{~s}$, and it can be tested that the model has certain practicability and rationality in the selection decision for the way out of the warehouse. It can provide a reference for the choice of a batch order outbound mode.

\section{Conclusion}

Based on the actual operation of the logistics distribution center, this paper analyzes and explores the inefficiency of the three-dimensional warehouse in the actual operation of the logistics. It is found that the key to solving the outbound efficiency of the three-dimensional warehouse area is how to effectively reduce the outbound storage. After the operation and comprehensive analysis of various methods to reduce the working time, it was found that the combination of EIQ analysis and the establishment of the judgment model is the most suitable method to solve this problem. Therefore, when selecting the problem of the three-dimensional warehouse shipping mode, the statistics of a large number of outbound and outbound orders are collected and collected, and a discriminant model based on the EIQ analysis is established. It is verified that the error calculated by the model is less than $\pm 25 \mathrm{~s}$ in the time difference of the outbound method compared with the data obtained from the actual experience. It can be proved that the model has certain practicality in the selection decision for the outbound mode. Sexuality and rationality can provide a reference for the selection of a batch order delivery method. As a result, the warehouse outbound efficiency and the service quality of the enterprise are improved, and the company is saved more costs. Therefore, from a comprehensive perspective, this research program can provide a certain reference value for the solution of the actual problems of enterprises.

\section{Conflicts of Interest}

The authors declare no conflicts of interest regarding the publication of this paper. 


\section{References}

[1] Li, Q.L. (2006) Research and Application of Sorting Strategies for Logistics Centers. Shandong University, Jinan.

[2] Li, J. (2009) Research on Configuration and Optimization of Automatic Sorting System in Cigarette Distribution Center. Shandong University, Jinan.

[3] Liu, J. (2010) Application of EIQ Analysis Method in Cigarette Distribution Center Planning. Beijing University of Posts and Telecommunications, Beijing.

[4] Chen, Y. (2015) ZH Power Supply Enterprise Emergency Material Reserve Strategy Research. South China University of Technology, Guangzhou.

[5] Fan, Q.Y. (2004) Research on Sorting System of Finished Tobacco Distribution Center. Kunming University of Science and Technology, Kunming.

[6] Li, F. (2009) Research on Replenishment Scheduling and Automatic Sorting Algorithm in Tobacco Distribution Center. Central South University, Changsha.

[7] Wu, Y. (2011) Application of Linear Programming Based on Greedy Algorithm in Optimization of Freight Location. Shanghai Jiaotong University, Shanghai. 